\title{
On evaluation of the warranty cost of a product with stochastic dependence between components
}

\author{
Nan Zhang ${ }^{\mathrm{a}}$, Mitra Fouladirad ${ }^{\mathrm{a}, *}$, Anne Barros $^{\mathrm{b}}$ \\ ${ }^{a}$ ICD-LM2S, Université de Technologie de Troyes, Troyes, France \\ ${ }^{b}$ Norwegian University of Science and Technology, Trondheim, Norway
}

\begin{abstract}
This paper analyses the expected warranty costs from the perspectives of the manufacturer and the consumer respectively. Both the non-renewing free replacement policy and the renewing replacement policy are examined regarding a two-component series system with stochastic dependence between components. It is assumed that whenever component 1 fails, a random damage to component 2 is induced whereas a component 2 failure causes the failure of component 1 . Component 2 fails when its total damage exceeds a pre-determined level $L$. By considering the consumer's behavior the product service time, the warranty costs allocations between the manufacturer and the consumer are presented. Numerical and Monte Carlo examples are given to demonstrate the applicability of the methodology. It is shown that, independent of the type of the warranty policy, the failure interaction between components has impact on the manufacturer profits and the consumer costs. The initial warranty length has impact on the product quality preferences to both the consumer and the manufacturer.
\end{abstract}

Keywords: Free replacement warranty; Two-component systems; Failure interaction; Virtual age; Imperfect repair

\section{Introduction}

A warranty is a contract between the manufacturer and the buyer[14]. It requests the manufacturer to repair, replace the product or to compensate

\footnotetext{
*Corresponding author

Email address: mitra.fouladirad@utt.fr (Mitra Fouladirad)
} 
the buyer if the product fails before a pre-determined time period, which is referred to as warranty period. Due to the development of technology and the intense competition between enterprises, warranty is more and more important and a lot of attention has been paid to it by both buyers and manufacturers. From the buyer's point of view, warranty is protectional which supports them to mitigate or even avoid losses if the item is out of function when properly used. Also, warranty is informational as the buyers generally deduce an item with longer warranty is more reliable and has better quality. From the manufacturer's point of view, warranty is also protectional and promotional because the conditional warranty prevents the misuse of products (for example, a broken-down HTC mobile phone within warranty period is not supposed to be repaired if the failure is caused by rough handling, exposure to moisture, dampness, extreme thermal, etc.) and can be seen as an advertisement since the buyers believe a longer warranty is more reliable when other circumstances are settled. Besides, warranty is an instrument which can be used in competitions with other manufacturers.

Basically, there are two common types of warranty policies in the literature: the free replacement/repair warranty $(\mathrm{FRW})([9,20,22,30])$ and the pro rata warranty $(\mathrm{PRW})[8,20]$. The former indicates that the manufacturer covers the total cost of repair or replacement of the product before the expiration of the warranty and the latter means that within the warranty period, a failed product is repaired at a cost which is proportional to the product age at failure. For example, Yun et al. [32] studied the optimal burn-in time to minimize the manufacturer's expected total cost including burn-in cost and the cumulative FRW cost. Wu et al. [29] developed a model for the burn-in time and warranty period optimization of non-repairable systems under FRW/PRW policy. Park et al. [21] examined the warranty of multi-component systems regarding the fixed warranty period and imperfect repair. There are many extensions and variations of warranty policies in the literature[2, 4].

In recent decades, in the warranty study, more and more focuses have been shifted from the single component system to the multi-component system. Compared to the single component systems, the reliability and cost analysis of multi-component systems are more appealing and more challenging. On the one hand, it provides more flexibility to model the system in terms of its reliability. For instance, some components can be modeled by their lifetimes [27], some can be characterized by their degradations $[3,33]$. Besides, it permits the 
manufacturer to benefit from the implementation of opportunistic maintenance to the product within the warranty period [1]. However, on the other hand, many problems are emerged consequently along with the advantages of multi-component systems. It requires the manufacture to develop warranty policies at the system level as well as at the component level. Therefore it is necessary to understand the contribution of each component to the system and the dependency among components. In which context, failure dependence between components is a major challenge which should be taken into account in the study of multi-component systems. In the literature, failure dependence was first presented by Murthy et al. ([15],[16]) in which they proposed two types of dependency in two-component and multi-component systems. For two-component systems, type I failure interaction indicates that the failure of component $i$ may act as a shock and cause the failure of the other one with probability $p_{i}, 0<p<1, i=1,2$. Type II failure interaction implies that other than inducing instantaneous failure, the failure of a component increases the failure rate of the surviving one. Type III failure interaction is introduced by Nakagawa and Murthy [18] which implies that component 1 failure causes a random damage to component 2 and component 2 failure induces the failure of component 1 . More and more maintenance and reliability analysis are developed since then, see for instance $([24,25,28,31])$.

It is noticed that in the previously reviewed studies, most of the warranty cost analysis dealt with the single-item system or the multi-item system with the assumption of consisting of independent items. In the framework of multi-component systems studies, the objectives are either maintenance-costoriented or system reliability/availability-oriented without considering the maintenance cost allocation between, for example, the product supplier and its consumer. Liu et al. [12] studied the warranty cost of multi-component systems with type I failure interaction between components under the renewing free replacement policy. The manufacturer's expected warranty costs with different system configurations have been examined.

In our study, we consider the type III failure interaction of a two-component system.

We intend to develop a modeling framework that is generic enough to have theoretical and methodological added value and precise enough to be meaningful for several applications. Generally speaking, in many technical systems, the failure of one component (component 1) can contribute to produce shocks/damages 
while another part can experience the shocks/damages or the consequences of these shocks/damages (component 2). It is often the case for mechanical or electrical systems, when one component failure ends in unexpected vibrations, frictions, overheating. For instance, in the break system, the disc rotor bolted to the wheel hub that rotates with the wheel and the brake pad made of steel backing plate are vital components. It is the fraction created by pushing the brake pad into the rotor which slows an automobile or brings it to a sudden halt. Whenever the break is carried out, fluid pressure forces the pads to against the disc. Adhesion mutual impact between the motional rotor and the static brake pad may cause wear at the surface of the brake pad. Besides, the surface of the rotor may be left rough due to the deformation of the metal. In a previous study, Nakagawa et al. [18] proposed a two component system with stochastic dependence where component 1 failure causes accumulative damages to component 2. Component 2 is considered as failure when its total damage exceeds a predetermined threshold. They discussed the optimization of the average long-run maintenance cost with respect to the failure number of component 1. In [23], likewise, the optimal maintenance cost of a similar problem was taken into consideration where they took the damage threshold and the system age as indicators. However, both of them considered the failure of component 2 as the effect of the damages due to the failure of component 1 . However, it is clear that in the brake system as we mentioned, the disk rotor may worn out due to its natural deterioration with respect to its age. Therefore, in this study, we consider the two-component system with failure interaction where component 2 undertakes its natural deterioration as well the damages generated by the failure of component 1.

The paper is organized as follows. The system descriptions, the hypotheses and the failure interactions between components are introduced in section 2. The warranty costs and profits from the perspectives of the manufacturer and the consumer are formulated in section 3. Section 4 is devoted to exact numerical calculations, Monte Carlo simulations and system sensitivity analysis. Finally, we make conclusions and give future extensions of our work in section 5 . 


\section{Model description}

First, a brief introduction about the virtual age method adopted in our model assumptions is given.

Virtual age (or effective age) depicts the real condition of the system other than the physical time elapsed from its new state. It is general enough which allows that the age of the repaired unit can be any value between 0 (renewal or perfect repair) and the age just before the failure (minimal repair). For instance, roughly speaking, a car can be considered as in the as-good-as-new state if we change its motor; or in the as-bad-as-old state if we change its battery. Kijima et al. [10,11] developed two imperfect maintenance models where they proposed the virtual age reduction method to describe the impact of repair. Let $B_{n}, A_{n}, X_{n}$ be respectively the component effective age after the $n$th repair, the component's $n$th repair degree and the time interval between the $(n-1)$ st and the $n$th repair. It is assumed in model 1 (Kijima model 1) that the repair reduces only the damage caused during the last survival period: $B_{n}=B_{n-1}+A_{n} X_{n}, B_{0}=0,0 \leq A_{n} \leq 1$. In Kijima model 2, damages induced before the $n$th repair are reduced: $B_{n}=A_{n}\left(B_{n-1}+X_{n}\right), B_{0}=0,0 \leq$ $A_{n} \leq 1$.

In this study, the Kijima model 1 is considered and we assume that the repair degree is independent of the failure number $n$ which yields $A_{n}=a$, $0 \leq a \leq 1$. The repair degenerates to minimal repair if $a=1$ and to perfect repair if $a=0$.

The system descriptions and the maintenance behaviors are given as following.

- Component 1 and component 2 are in series.

- Component 1 is repairable with lifetime distribution $F(\cdot)$, where $F(0)=$ 0 . It is imperfectly repaired when failure occurs. Let $X_{i}$ and $a$ denote the inter-maintenance time between the $(i-1)$ st and the $i$ th repair and the imperfect maintenance degree respectively. Then the virtual age of component 1 after the $i$ th repair is $B_{i}=B_{i-1}+a X_{i}, i=1,2, \cdots$ where $B_{0}=0$ and $0 \leq a \leq 1$.

- Whenever component 1 failure occurs, it causes a random amount of damage to component 2 . Let $Z_{j}(j=1,2, \ldots)$ be the damages which are 
independently and identically distributed random variables with distribution function $H(\cdot), H(0)=0$. Damages are additive and the total damage induced by component 1 by time $t$ is the sum of $Z_{j}$ occurred by time $t$.

- Component 2 is non-repairable and it fails when its damage level exceeds a pre-determined threshold $L, L>0$. The failure of component 2 induces the failure of component 1 . The system is replaced at the failure of component 2 .

- Denoted by $\{Y(t), t \geq 0\}$ the component 2 intrinsic damage level at time $t$ and $\sigma_{L}$ the natural arrival time or first exceeding time of the damage level $L$. Then without considering the damage induced by component 1 failure, the distribution function of $\sigma_{L}$ is

$$
G_{\sigma_{L}}(t)=\mathbb{P}\left\{\sigma_{L} \leq t\right\}=\mathbb{P}\{Y(t) \geq L\}, t \geq 0
$$

- Both the component repair times and the system renewal times are negligible.

Denoted $N(t)$ the component 1 failure number by time $t, V_{n}(\cdot)$ the distribution function of $B_{n}$, and $p_{n}(t)=\mathbb{P}(N(t)=n)$. According to [10],

$$
\bar{V}_{n+1}(x)=\bar{V}_{n}(x)+\int_{0}^{x} \frac{\bar{F}\left(y+\frac{x-y}{a}\right)}{\bar{F}(y)} \mathrm{d} V_{n}(y)
$$

the following equation can be easily derived.

$$
\begin{aligned}
p_{n}(t) & =\mathbb{P}\left(\sum_{i=1}^{n} X_{i} \leq t<\sum_{i=1}^{n+1} X_{i}\right)=\mathbb{P}\left(\frac{B_{n}}{a} \leq t<\frac{B_{n+1}}{a}\right) \\
& =\int_{0}^{a t} \frac{\bar{F}\left(y+\frac{a t-y}{a}\right)}{\bar{F}(y)} v_{n}(y) \mathrm{d} y
\end{aligned}
$$

where $\bar{F}(\cdot)=1-F(\cdot), v_{n}(x)=\frac{d}{d x} V_{n}(x), v_{1}(x)=\frac{1}{a} f\left(\frac{x}{a}\right)$ and $v_{n+1}(x)=$ $\frac{1}{a} \int_{0}^{x} \frac{f\left(y+\frac{x-y}{a}\right)}{\bar{F}(y)} v_{n}(y) d y$ for $n \geq 1[10]$.

Besides, the component 2 lifetime distribution $F_{s}(t)$ is presented in the following proposition. 
Proposition 1. The component 2 lifetime distribution $F_{s}(t)$ is given by

$$
F_{s}(t)=p_{0}(t) G_{\sigma_{L}}(t)+\sum_{k=1}^{\infty} p_{k}(t) \int_{0}^{\infty} G_{\sigma_{L-z}}(t) \mathrm{d} H^{*(k)}(z)
$$

where $p_{k}(t)$ is the probability mass function of the component 1 failure number given in equation (3). $H(\cdot)$ is the damage distribution function and $H^{*(k)}(t)$ is the $k$-fold convolution of $H(t)$ with itself.

Proof. Let $T_{i}, i=1,2, \cdots$, be the time interval of the $i$ th system renewal time which are identically and independently distributed. Assume that the distribution function is $F_{s}(t)$. Then

$$
\begin{aligned}
F_{s}(t)=\mathbb{P}\left\{T_{1} \leq t\right\} & =p_{0}(t) \mathbb{P}(Y(t)>L)+\sum_{k=1}^{\infty} \mathbb{P}\left(Y(t)+\sum_{i=1}^{k} Z_{i}>L \mid N(t)=k\right) \mathbb{P}(N(t)=k) \\
& =p_{0}(t) \mathbb{P}(Y(t)>L)+\sum_{k=1}^{\infty} p_{k}(t) \int_{0}^{\infty} \mathbb{P}(Y(t)>(L-z)) \mathrm{d} H^{*(k)}(z) \\
& =p_{0}(t) G_{\sigma_{L}}(t)+\sum_{k=1}^{\infty} p_{k}(t) \int_{0}^{\infty} G_{\sigma_{L-z}}(t) \mathrm{d} H^{*(k)}(z)
\end{aligned}
$$

where $G_{\sigma_{x}}(t)=1$ when $x<0$. Therefore the component 2 lifetime distribution function is obtained. $\diamond$

\section{Warranty analysis}

In this section, we intend to consider two types of warranty policies which are the non-renewing free replacement policies and the renewing free replacement policy (we will call them the non-renewing FRW and the RFRW for short hereafter). More precisely, it means, during the initial warranty period $W$,

- under the non-renewing FRW

- component 1 is imperfectly repaired at its failure at time $T_{f 1}$ and the warranty of the system remains valid during the remaining $W-T_{f 1}$ period.

- the system is replaced at the failure of component 2 at time $T_{f 2}$ and the warranty of the system remains valid only during the remaining $W-T_{f 2}$ period. 
- under the RFRW,

- component 1 is imperfectly repaired at its failure at time $T_{f 1}$ and the warranty of the system remains valid during the remaining $W-T_{f 1}$ period.

- the system is replaced at the failure of component 2 at time $T_{f 2}$ and a full system warranty period of length $W$ is provided.

Suppose that to the manufacturer, the component 1 repair cost and the system replace cost are $c_{1}$ and $c_{2}$ respectively. First, under the two warranty policies, from the perspective of the manufacturer, the expected warranty costs are formulated.

\subsection{The manufacturer's expected warranty costs}

For simplicity, we call the system failure as minor failure if it contains only component 1 failure and major failure if both the two components in the system fail.

\subsubsection{The expected manufacturer's cost under the non-renewing FRW}

Under the non-renewing FRW, the manufacturer covers the repair or replacement cost up to a duration $W$ from the initial product purchase time. Denote $\mathbb{E}(C(W))$ be the manufacturer's expected warranty cost under nonrenewing FRW within warranty period $W$. The following theorem gives the expected manufacturer cost under non-renewing FRW.

Theorem 3.1. Under the non-renewing FRW, the expected manufacturer cost $\mathbb{E}(C(W))$ within the warranty period $W$ is given by:

$$
\mathbb{E}(C(W))=\int_{0}^{W}[1+M(W-t)] k(t) \mathrm{d} t
$$

where $M(t)$ is the renewal function related with $F_{s}(t)$ which can be given by $M(t)=\sum_{n=1}^{\infty} F_{s}^{(n)}(t), k(t)=c_{1} \bar{F}_{s}(t) \sum_{n=0}^{\infty} n\left[a v_{n}(a t)-\int_{0}^{a t} \frac{f\left(y+\frac{a t-y}{a}\right)}{\bar{F}(y)} v_{n}(y) \mathrm{d} y\right]+$ $c_{2} f_{s}(t), F_{s}(t)\left(f_{s}(t)\right), F(t)(f(t))$ are the lifetime distribution (density) functions of component 2 and component 1 respectively, $\bar{F}_{s}(t)=1-F_{s}(t)$, a is the imperfect maintenance degree of component $1, v_{n}(t)$ is given in equation 3. 
Proof. By conditioning on the first renewal time of the system $Y_{T_{1}}$, we have:

$$
\mathbb{E}\left(C(W) \mid Y_{T_{1}}=t\right)= \begin{cases}c_{1} \mathbb{E}(N(W)) & t>W \\ c_{2}+c_{1} \mathbb{E}(N(t))+\mathbb{E}(C(W-t)) & t \leq W\end{cases}
$$

where $\mathbb{E}(N(t))=\sum_{n=0}^{\infty} n p_{n}(t)$ is the expected maintenance times of component 1 in $[0, t]$ before system replacement. Based on the law of total probability:

$$
\begin{aligned}
\mathbb{E}(C(W)) & =c_{1} \bar{F}_{s}(W) \mathbb{E}(N(W))+\int_{0}^{W}\left\{c_{2}+c_{1} \mathbb{E}(N(t))+\mathbb{E}(C(W-t))\right\} \mathrm{d} F_{s}(t) \\
& =K(W)+\int_{0}^{W} \mathbb{E}(C(W-t)) \mathrm{d} F_{s}(t)
\end{aligned}
$$

where $K(W)=c_{1} \bar{F}_{s}(W) \mathbb{E}(N(W))+\int_{0}^{W}\left\{c_{2}+c_{1} \mathbb{E}(N(t))\right\} \mathrm{d} F_{s}(t)$. From the renewal property, equation (6) is equal to

$$
\mathbb{E}(C(W))=K(W)+\int_{0}^{W} K(W-x) \mathrm{d} M(x)
$$

where $M(t)$ is the renewal function related with $F_{s}(t)$. The above equation is equal to

$$
\mathbb{E}(C(W))=\int_{0}^{W}[1+M(W-t)] k(t) \mathrm{d} t
$$

where $k(t)=\frac{\mathrm{d} K(t)}{\mathrm{d} t}=c_{1} \bar{F}_{s}(t) \sum_{n=0}^{\infty} n\left[a v_{n}(a t)-\int_{0}^{a t} \frac{f\left(y+\frac{a t-y}{a}\right)}{\bar{F}(y)} v_{n}(y) \mathrm{d} y\right]+c_{2} f_{s}(t)$. $\diamond$

In our study, only the expected warranty cost of the manufacturer within the warranty period is considered. Meanwhile, as a competition means, warranty plays an active role in promoting the market share as well as the profitability of the manufacturer. Therefore, it is necessary to compare the marginal maintenance cost and the marginal warranty gains in developing warranty policies. A lower limit of the marginal maintenance cost is given in the following remark.

Remark 1: Under the non-renewing FRW, the marginal maintenance cost $M^{\text {non }}(W)$ follows the following inequality.

$$
M^{n o n}(W) \geq k(W)
$$




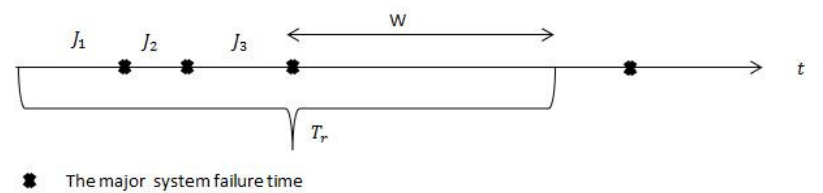

Figure 1: A possible system warranty period under RFRW policy

where $k(W)$ is given in Theorem 3.1. Particularly, when component 1 is minimally repaired at failure $(a=1), k(W)$ can be simplified to

$$
k(W)=c_{1} \bar{F}_{s}(W) r(W)+c_{2} f_{s}(W)
$$

where $r(W)$ is the failure rate of component 1 defined as $r(W) \mathrm{d} W=$ $\frac{\mathrm{d} F(W)}{F(W)}, F_{s}(W)$ is given in equation (4).

\subsubsection{The expected manufacturer's cost under renewing FRW policy}

Under the RFRW, the major failure within the warranty period is replaced by a new one (repaired as good as new) with a full system warranty period $W$. Let us denote $T_{r}$ the warranty cycle which is a time interval from the system purchase time until the expiration of the warranty. It is obvious that in this situation the warranty cycle depends on the initial warranty period $W$, the system renewal times and the time interval between two consecutive system renewal times. Denote $N_{r}(W)$ be the system renewal times when the initial warranty period is $W$, and $J_{i}, i=1,2, \cdots, N_{r}(W)$ the corresponding time interval between the $(i-1)$ th and the $i$ th system renewal time which are identically and independently distributed random variables. Figure 1 gives an example of total system warranty length under RFRW. It can be noticed that:

$$
T_{r}=J_{1}+J_{2}+\cdots+J_{N_{r}(W)}+W
$$

Let $\mathbb{E}\left(C^{R}(W)\right)$ be the expected manufacturer warranty cost under RFRW. We have the following theorem.

Theorem 3.2. Under the RFRW, the expected system cost is

$$
\mathbb{E}\left(C^{R}(W)\right)=\frac{c_{2} F_{s}(W)+c_{1} \int_{0}^{W} \mathbb{E} N(t) \mathrm{d} F_{s}(t)}{1-F_{s}(W)}+c_{1} \mathbb{E} N(W)
$$


where $F_{s}(t)$ is the component 2 lifetime distribution function, $\bar{F}_{s}(t)=1-$ $F_{s}(t), \mathbb{E}(N(t))=\sum_{n=1}^{\infty} n p_{n}(t)$ which is the expected failure times of component 1 before system failure.

Proof. It can be noticed that $\mathbb{P}\left(N_{r}(W)=n\right)=F_{s}^{n}(W) \bar{F}_{s}(W)$. Denote $F_{J_{i}}(t)$ the distribution function of $J_{i}$, then

$$
\begin{aligned}
F_{J_{i}}(t) & =\mathbb{P}\left(J_{i} \leq t\right)=\mathbb{P}\left(T_{i} \leq t \mid T_{i} \leq W\right) \\
& =\left\{\begin{array}{cl}
\frac{F_{s}(t)}{F_{s}(W)} & \text { if } t<W \\
1 & \text { if } t \geq W
\end{array}\right.
\end{aligned}
$$

Let $C_{n W}(T)$ be the total system warranty cost by time $T$ under RFRW with warranty period $W$ given that the number of major system failure $n$. Then

$$
\begin{aligned}
\mathbb{E}\left(C^{R}(W)\right)= & \mathbb{E}\left[\mathbb{E}\left(C^{R}\left(W \mid N_{r}(W)=n\right)\right)\right] \\
= & \sum_{n=0}^{\infty} F_{s}^{n}(W) \bar{F}_{s}(W) \mathbb{E}\left[C_{n W}\left(J_{1}+J_{2}+\cdots+J_{n}+W\right)\right] \\
= & \sum_{n=0}^{\infty} F_{s}^{n}(W) \bar{F}_{s}(W) \\
& \int_{0}^{W} \cdots \int_{0}^{W}\left(n c_{2}+c_{1} \sum_{i=1}^{n} \mathbb{E}\left(N\left(j_{i}\right)\right)+c_{1} \mathbb{E}(N(W)) \mathrm{d} F_{J_{1}}\left(j_{1}\right) \cdots \mathrm{d} F_{J_{n}}\left(j_{n}\right)\right. \\
= & \sum_{n=0}^{\infty} \bar{F}_{s}(W)\left(n c_{2} F_{s}^{n}(W)+c_{1} \mathbb{E}(N(W)) F_{s}^{n}(W)+n c_{1} F_{s}^{n-1}(W) \int_{0}^{W} \mathbb{E}(N(t)) \mathrm{d} F_{s}(t)\right) \\
= & \sum_{n=0}^{\infty} \bar{F}_{s}(W) F_{s}^{n}(W)\left(n c_{2}+c_{1} \mathbb{E}(N(W))+n c_{1} \frac{\int_{0}^{W} \mathbb{E}(N(t)) \mathrm{d} F_{s}(t)}{F_{s}(W)}\right) \\
= & \frac{c_{2} F_{s}(W)+c_{1} \int_{0}^{W} \mathbb{E} N(t) \mathrm{d} F_{s}(t)}{1-F_{s}(W)}+c_{1} \mathbb{E} N(W)
\end{aligned}
$$

Therefore we obtain the warranty cost under RFRW for the manufacturer. $\diamond$

Remark 2:If $F$ is IFR, when component 1 is minimally repaired, the following inequalities regarding the marginal maintenance cost $M^{r e}(W)$ are valid.

$$
\begin{aligned}
& M^{r e}(W) \geq \frac{c_{2} f_{s}(W)+c_{1} \Lambda(W) f_{s}(W)\left(1-F_{s}(W)\right)}{\left(1-F_{s}(W)\right)^{2}}+c_{1} r(W) \\
& M^{r e}(W) \leq \frac{c_{2} f_{s}(W)+c_{1} \Lambda(W) f_{s}(W)}{\left(1-F_{s}(W)\right)^{2}}+c_{1} r(W)
\end{aligned}
$$


Proof. Let be $r(t)$ the failure rate of component 1 by time $t$. The failure arrives according to a non-homogeneous Poisson process when component 1 is minimally repaired which yields:

$$
\mathbb{E}(W)=\Lambda(W)
$$

where $\Lambda(W)=\int_{0}^{W} r(t) \mathrm{d} t$. Therefore

$$
\begin{aligned}
\left.\frac{\mathrm{d} \mathbb{E}\left(C^{R}(W)\right.}{\mathrm{d} W}\right)= & \frac{c_{2} f_{s}(W)+c_{1} \Lambda(W) f_{s}(W)\left(1-F_{s}(W)\right)}{\left(1-F_{s}(W)\right)^{2}} \\
& +\frac{c_{1} \int_{0}^{W} \mathbb{E} N(W) \mathrm{d} F_{s}(W)}{\left(1-F_{s}(W)\right)^{2}}+c_{1} r(W) \\
& \geq \frac{c_{2} f_{s}(W)+c_{1} \Lambda(W) f_{s}(W)\left(1-F_{s}(W)\right)}{\left(1-F_{s}(W)\right)^{2}}+c_{1} r(W)
\end{aligned}
$$

Particularly, when $\boldsymbol{F}$ is IFR,

$$
\int_{0}^{W} \mathbb{E} N(t) \mathrm{d} F_{s}(t) \leq \mathbb{E} N(W) F_{s}(W)
$$

which yields

$$
\left.\frac{\mathrm{d} \mathbb{E}\left(C^{R}(W)\right.}{\mathrm{d} W}\right) \leq \frac{c_{2} f_{s}(W)+c_{1} \Lambda(W) f_{s}(W)}{\left(1-F_{s}(W)\right)^{2}}+c_{1} r(W)
$$

$\diamond$

By now, we have formulated the expected warranty costs of the manufacturer under the non-renewing FRW and the RFRW. One step further, if we take the consumer's behavior into consideration and assume that he/she intends to put the product in service until $T$, it follows that $W<T<\infty$. After this, the system is not repaired when failure occurs. Besides, after the product warranty, the consumer chose coming back to the original manufacturer (or the supplier, retailer, seller etc. here we do not distinguish them) for the maintenance. Denoted $c_{11}, c_{22}$ are the component 1 repair price and the system renewal price respectively to the consumer. It is rational to set $c_{i i}>c_{i}, i=1,2$ as the manufacturer earnings from the difference between the prices $c_{11}, c_{22}$ and the corresponding costs $c_{1}, c_{2}$. In section 3.2 , the warranty cost and profit to the consumer and the manufacturer respectively are examined. 


\subsection{The expected warranty profit and cost in $(0, T]$}

In the following, by assuming that the product service time is $T, W<$ $T<\infty$. The warranty cost analysis of different warranty policies in $(0, T]$ are explored. Hereafter we assume that component 1 is minimally repaired when failure occurs.

\subsubsection{The expected warranty profit and cost in $(0, T]$ under the $R F R W$}

Let $C^{s}(T)$ and $T P^{s}(T)$ be the total warranty cost of the consumer (owner) and the profit of the manufacturer respectively, let $\mathbb{E}\left(C^{s}(T)\right)$ and $\mathbb{E}\left(T P^{s}(T)\right)$ be their expectations. They are formulated in the following theorem.

Theorem 3.3. Under the RFRW, the expected maintenance cost of the consumer and the expected total profit of the manufacturer in $[0, T]$ are as follows:

$$
\begin{aligned}
\mathbb{E}\left(C^{s}(T)\right) & =\int_{W}^{T}(1+M(T-u)) \mathrm{d} h(u) \\
\mathbb{E}\left(T P^{s}(T)\right) & =\mathbb{E}\left(C^{s}(T)\right)-\mathbb{E}\left(C^{1}(T)\right)-c_{2} M(T)
\end{aligned}
$$

where

$$
\begin{aligned}
h(T) & =c_{22}\left(F_{s}(T)-F_{s}(W)\right)+c_{11} \int_{W}^{T} \bar{F}_{s}(t) \mathrm{d} \mathbb{E} N(t) \text { for } T \geq W \\
\mathbb{E}\left(C^{1}(T)\right) & =h_{1}(T)+\int_{0}^{T} h_{1}(T-t) \mathrm{d} M(t) \\
h_{1}(T) & =c_{1} \mathbb{E}(N(T))-c_{1} \int_{0}^{T} F_{s}(t) \mathrm{d} \mathbb{E}(N(t))
\end{aligned}
$$

$M(t)=\sum_{n=1}^{\infty} F_{s}^{(n)}(t)$ is the system renewal function related with $F_{n}(t)$ and $\mathbb{E} N(t)=\sum_{n=1}^{\infty} n p_{n}(t)$ is the expected failure number of component 1 in $[0, t]$ before system replacement. $F_{s}(t)\left(f_{s}(t)\right), F(t)(f(t))$ are the lifetime distribution (density) functions of component 2 and component 1 respectively, $\bar{F}_{s}(t)=1-F_{s}(t)$.

The proof is omitted as it is similar to the proof the Theorem 3.1. 
3.2.2. The expected warranty profit and cost under the non-renewing FRW

Under the non-renewing FRW, the manufacturer pays the full warranty cost if the system fails within the warranty period and the warranty is not renewed. It is reasonable to assume that the consumer is provided with a new system with a full warranty if the major failure occurs in $(W, T]$ and he/she covers himself/herself the full purchase cost. Here we adopt the method proposed by Nguyen and Murthy [19] for the expected warranty cost calculation.

Let $C^{s n}(T)$ and $T P^{s n}(T)$ be the total warranty cost of the consumer and the profit of the manufacturer respectively. Let $\mathbb{E}\left(C^{s n}(T)\right), \mathbb{E}\left(T P^{s n}(T)\right)$ be their expectations.

Theorem 3.4. Under the non-renewing FRW, when component 1 failures occur according to a Poisson process, the expected warranty cost of the consumer and the expected total profit of the manufacturer in $[0, T]$ are derived as follows:

$$
\begin{aligned}
\mathbb{E}\left(C^{s n}(T)\right) & =h_{n}(T)+\int_{0}^{T} h_{n}(T-u) \mathrm{d} M_{U}(u) \\
\mathbb{E}\left(T P^{s n}(T)\right) & =\mathbb{E}\left(C^{s n}(T)\right)-c_{1} \mathbb{E} N(T)-c_{2} M(T)
\end{aligned}
$$

where

$$
\begin{aligned}
F_{U}(t) & =F_{s}(t)-\int_{0}^{W} \bar{F}(t-x) \mathrm{d} M(x) \quad \text { for } t \geq W \text { and } 0 \text { otherwise } \\
h_{n}(t) & =c_{22} F_{U}(t)+c_{11} \int_{W}^{t} \bar{F}_{U}(\theta) \mathrm{d} \mathbb{E} N(\theta) \quad \text { for } t \geq W \text { and } 0 \text { otherwise }
\end{aligned}
$$

$M_{U}(t)(M(t))$ is the system renewal function related with $F_{U}(t)\left(F_{n}(t)\right)$ and $\mathbb{E} N(t)=\sum_{n=1}^{\infty} n p_{n}(t)$ is the expected failure number of component 1 in $[0, t]$ before system replacement. $F_{s}(t)\left(f_{s}(t)\right), F(t)(f(t))$ are the lifetime distribution (density) functions of component 2 and component 1 respectively, $\bar{F}_{s}(t)=1-F_{s}(t)$.

Proof. Let $U(U>W)$ be the time interval between two consecutive system purchase time by the consumer. That is to say that the system renewal cost are covered by the consumer rather than the manufacturer. Denote $\gamma(W)$ be the residual life to the system at time $W$, then $U=W+$ $\gamma(W)$. Let $F_{\gamma}(t), F_{U}(t)$ be the distribution function of $\gamma(W)$ and $U$ then from the renewal theory we know [17]:

$$
F_{\gamma}(t)=F_{s}(W+t)-\int_{0}^{W} \bar{F}_{s}(W+t-x) \mathrm{d} M(x)
$$




$$
E(U)=(M(W)+1) \int_{0}^{\infty} \bar{F}_{s}(t) \mathrm{d} t
$$

where $M(W)=\sum_{n=1}^{\infty} F_{s}^{(n)}(W)$ and

$$
F_{U}(t)= \begin{cases}0 & 0 \leq t \leq W \\ F_{\gamma}(t-W) & \mathrm{W}<t\end{cases}
$$

Thus by conditioning on the first purchase time of the consumer, his/her expected total cost can be derived. The expected profit of the manufacturer is then obtained as the revenue minus the maintenance cost by time $T$. $\diamond$

\subsection{The long run average costs and profits}

In industrial manufacturing, many systems are longevous and supposed to operate for a long time, which is the case $T=\infty$. In the following, we derive the system average warranty cost of the consumer and the average profit of the manufacturer considering the long-run time horizon $T=\infty$.

\subsubsection{No warranty}

In this case, the system repair and replacement costs are not covered by the manufacturer but are paid by the consumer. Suppose $C^{l}$ and $T P^{l}$ be respectively the average cost and the profit of the consumer and the manufacturer on the long time horizon.

Theorem 3.5. $C^{l}$ and $T P^{l}$ are given as follows

$$
\begin{aligned}
C^{l} & =\frac{c_{22}+c_{11} \int_{0}^{\infty} \mathbb{E} N(t) \mathrm{d} F_{s}(t)}{\int_{0}^{\infty} \bar{F}_{s}(t) \mathrm{d} t} \\
T P^{l} & =C^{l}-\frac{c_{2}+c_{1} \int_{0}^{\infty} \mathbb{E} N(t) \mathrm{d} F_{s}(t)}{\int_{0}^{\infty} \bar{F}_{s}(t) \mathrm{d} t}
\end{aligned}
$$

It can be easily proved by the renewal reward theorem. The proof is omitted here. Proof. The system operation process is seen as a renewal process since the system is renewed at component 2 failure. Let $\phi_{i}$, be the length of the $i$ th replacement cycle and $R_{i}$ the cost over $\phi_{i}, i=1,2, \cdots$. Then $\left\{\phi_{i}, R_{i}\right\}$ constitutes a renewal reward process which yields

$$
C^{l}=\frac{\mathbb{E}\left(\phi_{1}\right)}{\mathbb{E}\left(R_{1}\right)}
$$


where $\mathbb{E}\left(\phi_{1}\right), \mathbb{E}\left(R_{1}\right)$ are the expectations of $\phi_{1}, R_{1}$ correspondingly. Since

$$
\begin{aligned}
& \mathbb{E}\left(\phi_{1}\right)=c_{22}+c_{11} \int_{0}^{\infty} \mathbb{E} N(t) \mathrm{d} F_{s}(t) \\
& \mathbb{E}\left(R_{1}\right)=\int_{0}^{\infty} \bar{F}_{s}(t) \mathrm{d} t
\end{aligned}
$$

the expected maintenance cost of the consumer without warranty is derived. Similarly the maintenance profit of the manufacturer can be calculated.»

\subsubsection{Under the RFRW policy}

Suppose that $C^{r}$ and $T P^{r}$ are respectively the average cost and the profit to the consumer and the manufacturer under RFRW, then

Theorem 3.6. $C^{r}$ and $T P^{r}$ are given as

$$
\begin{aligned}
C^{r} & =\frac{c_{22} \bar{F}_{s}(W)+c_{11} \int_{W}^{\infty}(\mathbb{E} N(t)-\mathbb{E} N(W)) \mathrm{d} F_{s}(t)}{\int_{0}^{\infty} \bar{F}_{s}(t) \mathrm{d} t} \\
T P^{r} & =C^{r}-\frac{c_{2}+c_{1} \int_{0}^{\infty} \mathbb{E} N(t) \mathrm{d} F_{s}(t)}{\int_{0}^{\infty} \bar{F}_{s}(t) \mathrm{d} t}
\end{aligned}
$$

The theorem is easily obtained by the renewal reward theorem as in Theorem 3.5 .

\subsubsection{Under the non-renewing FRW policy}

Suppose that component 1 failure occurs according to a Poisson process. Let $C^{n r}$ and $T P^{n r}$ be the expected average consumer cost and the manufacturer profit under non-renewing FRW.

Theorem 3.7. $C^{n r}$ and $T P^{n r}$ are given as

$$
\begin{aligned}
C^{n r} & =\frac{c_{22}+c_{11} \int_{W}^{\infty}(\mathbb{E} N(t)-\mathbb{E} N(W)) \mathrm{d} F_{U}(t)}{(M(W)+1) \int_{0}^{\infty} \bar{F}_{s}(t) \mathrm{d} t} \\
T P^{n r} & =C^{n r}-\frac{c_{2}(M(W)+1)+c_{1} \int_{W}^{\infty} \mathbb{E} N(t) \mathrm{d} F_{U}(t)}{(M(W)+1) \int_{0}^{\infty} \bar{F}_{s}(t) \mathrm{d} t}
\end{aligned}
$$

The theorem is easily obtained by the renewal reward Theorem. 


\section{Numerical examples}

\subsection{Parameter estimation}

Here we briefly provide an approach of the estimation of parameter settings in this study. To do with this, suppose that there are $M$ systems which are periodically observed at time $k \Delta$, $k=1,2, \cdots, N, T=N \Delta$. The degradation increment of component 2 of component $i$ at time $j \Delta$ is $y_{i j}, i=1,2, \cdots, M ; j=$ $1,2, \cdots, N$. Whenever component 1 failure occurs, it is replaced with an as-good-as-new one. Let be $x_{i k} \Delta$ the time at which the $k$ th failure of component $\mathbf{1}$ is observed in system $i, i=1,2, \cdots, M ; k \in N^{*}$. The collected data is the can be classified into three types:

- $A=\left\{x_{i k} \Delta-x_{i k-1} \Delta\right\}, i=1,2, \cdots, M ; k \in N^{*}$ are the lifetime data of component 1 which can be used in estimating parameters corresponding to the lifetime of component 1 ;

- $B=\left\{y_{i j} ; j \Delta \neq x_{i k} \Delta\right\}$ are the natural degradation data of component 2 in the period of time $\Delta$ which can be implemented in the parameter estimation of the lifetime of component 2;

- $C=\left\{y_{i j}\right.$; if $\left.y_{i j} \notin B\right\}$ are the summation of the natural degradation as well as the damage incurred to component 2 because of the failure of component 1 which can be implied in the estimation of the damage level.

An example is given in the following. Here we suppose that component 1 is Weibull distributed with lifetime distribution function $F(t)=1-e^{-\left(\frac{t}{\lambda}\right)^{b}}, t>0$. The damage induced to component 2 by component 1 failure has exponential distribution with expectation $\lambda$. The natural deterioration of component 2 follows a homogeneous Gamma process which has been extensively applied due to its successfully data-fitted property in describing system degradation associated to crack , corrosion, etc. [26]. The density function is

$$
g_{\alpha t, \beta}(u)=\frac{\beta^{\alpha t} u^{\alpha t-1} e^{-\beta u}}{\Gamma(\alpha t)}
$$

where

$$
\Gamma(\alpha)=\int_{0}^{\infty} u^{\alpha-1} e^{-u} d u
$$


It is clear that

$$
G_{\sigma_{L}}(t)=\frac{\Gamma(\alpha t, L \beta)}{\Gamma(\alpha t)}, t \geq 0
$$

where

$$
\Gamma(\alpha, x)=\int_{x}^{\infty} z^{\alpha-1} e^{-z} d z
$$

Therefore the parameters $a, b$ can be estimated by maximum likelihood function using dataset $A$; the scale parameter and the shape parameter of the gamma distribution can be derived with the dataset $B$. In this example, it is clear that the density function of the damage level $d(z)$ can be calculated by

$$
d(z)=\frac{\mathrm{d}}{\mathrm{d} z} \int_{0}^{z}\left(1-e^{-\mu(z-\theta)}\right) g_{\alpha \Delta, \beta}(\theta) \mathrm{d} \theta=\mu \int_{0}^{z} e^{\mu \theta} g_{\hat{\alpha} \Delta, \hat{\beta}}(\theta) \mathrm{d} \theta
$$

where $\hat{\alpha}$ and $\hat{\beta}$ are the estimation of $\alpha$ and $\beta$ in the previous step. Therefore the total log-likelihood is

$$
\ln \mathscr{L}\left(\mu ; \forall y_{i j} \in C\right)=P \ln (\mu)-\sum_{y_{i j} \in C} y_{i j}+\sum_{z_{i j} \in C} \ln \left(\int_{0}^{z_{i j}} e^{\mu \theta} g_{\hat{\alpha} \Delta, \hat{\beta}}(\theta)\right) \mathrm{d} \theta
$$

where $P$ is the cardinality of $C$ and the maximum likelihood estimator of $\lambda$ can be derived by

$$
\hat{\mu}=\underset{\mu}{\arg \max }\left(P \ln (\mu)-\sum_{y_{i j} \in C} y_{i j}+\sum_{z_{i j} \in C} \ln \left(\int_{0}^{z_{i j}} e^{\mu \theta} g_{\hat{\alpha} \Delta, \hat{\beta}}(\theta)\right) \mathrm{d} \theta\right)
$$

Table 1 presents the estimation results when $M=100$ and $\Delta=0.1$. Generally, the result is acceptable with so many samples $(M=100$

\begin{tabular}{cccc}
\hline Parameter & True value & Estimated value & $95 \%$ confidential interval \\
$\lambda$ & 1.5 & 1.5227 & {$[1.4276,1.6241]$} \\
$b$ & 1.2 & 1.2123 & {$[1.1437,1.2851]$} \\
$\alpha$ & 1 & 1.1790 & {$[1.1530,1.2050]$} \\
$\beta$ & 3 & 3.3422 & {$[3.1969,3.5336]$} \\
$\mu$ & 2 & 2.0833 & {$[1.9359,2.2307]$} \\
\hline
\end{tabular}

Table 1: Estimation results of system parameters with system numbers $M=100$ and observation interval $\Delta=0.1$ 
and the total replace number of component 1 in this simulation is 696). Besides, expert advice in reality may play a significant role to decide the parameter settings.

In the following, the warranty cost under, respectively, RFRW and nonrenewing FRW is presented first through exact numerical calculations and Monte Carlo simulations, and then using Monte Carlo simulations. The impact of system parameter setting associated to the warranty costs is explored. The consumer cost and the manufacturer profit under the two warranty policies are compared.

\subsection{Illustrative examples: cost calculation by two methods}

As an illustrative example, we assume that $a=10, b=2, \mu=1, \alpha=$ $4, \beta=2, c_{1}=50, c_{2}=250$ and $a=1$ which implies that component 1 undergoes minimal repair when failure occurs. From Theorems 3.1 and 3.2, the expected warranty costs under the non-renewing FRW and RFRW respectively are obtained respectively: $\mathbb{E}(C(W))=12.50$ where $W=5$ and $L=20$ and $\mathbb{E}\left(C^{R}(W)\right)=467.6$ where $W=10$ and $L=20$. The exact results are compared with Monte Carlo simulations which are more time consuming. In Figure $\mathbf{2}$ and Figure 3, the green line represents the theoretical result and the blue asterisks represent the simulation results with respect to the number of the simulations. It is seen that under both cases, the simulations calculations are convergent to the theoretical results when the number of the simulations is 20000 which indicated that the results under the two methods are coincident.

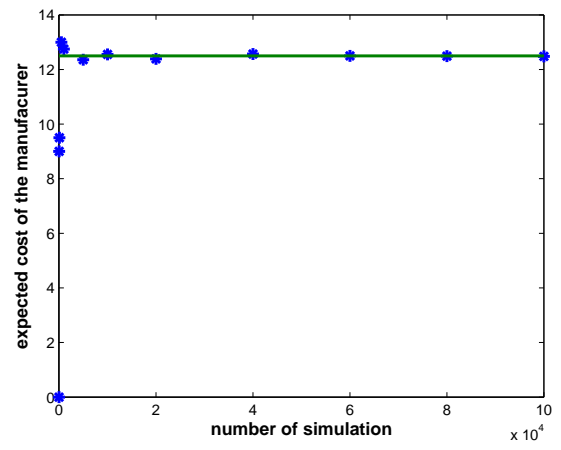

Figure 2: the total cost under non-renewing FRW

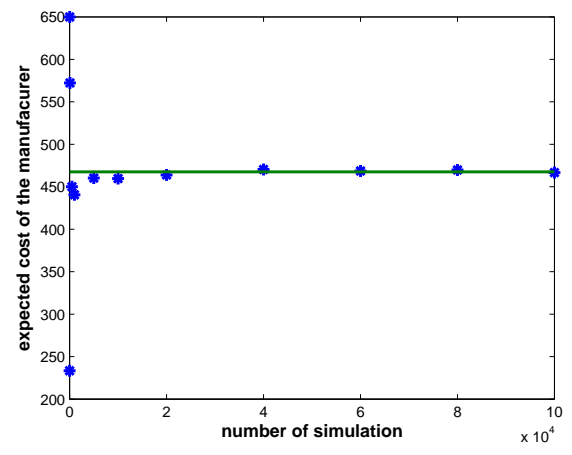

Figure 3: the total cost under RFRW 


\subsection{Sensitivity analysis}

First, let $S=\left\{\lambda=8, b=2, a=1, \mu=4, \alpha=4, \beta=2, L=20, c_{11}=\right.$ $\left.5, c_{22}=25, c_{1}=1, c_{2}=4, T=20\right\}$. In the following, the impacts of some parameters involved in the model are investigated by changing one parameter each time and comparing with the results derived by using the original data set $S$.

The impacts of the initial warranty length and the failure interaction

We first study the effects of the warranty period and the failure interaction to the manufacturer's profit and the consumer's cost respectively. One can expect the extension of the warranty period is more beneficial to the consumer and less profitable to the manufacturer. This latter is confirmed by Figures 4 and 5. Figure 4 shows the consumer's warranty costs and the manufacturer's profits under the RFRW with various values of $W$ and $\mu$. Whereas, Figure 5 illustrates the long run average warranty costs and profits correspondingly. As expected, the difference between the total expected manufacturer profit $\mathbb{E}\left(T P^{s}\right)$ and the expected cost of the consumer $\mathbb{E}\left(C^{s}\right)$ is constant.

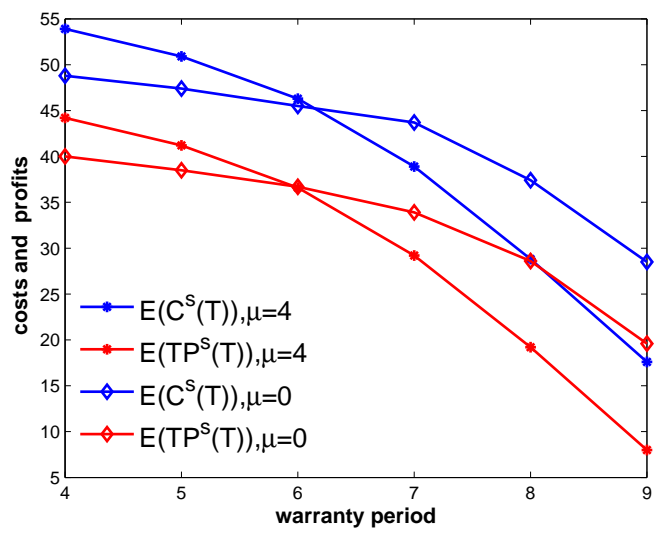

Figure 4: The expected consumer cost and manufacturer profit under RFRW with various values of $W$ and $\mu$ 


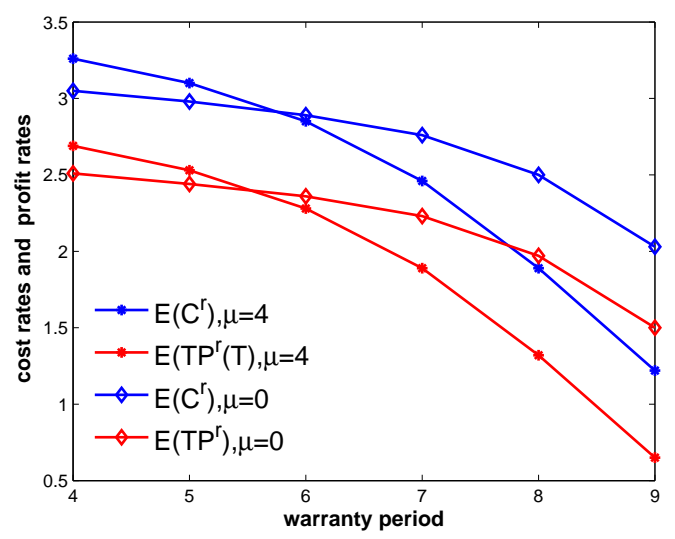

Figure 5: The long run average consumer cost and manufacturer profit under RFRW with different values of $W$ and $\mu$.

In both cases of short-run and long-run horizon, the expected warranty cost of the consumer (or the expected profit of the manufacturer) is a decreasing function of the warranty period. It is easy to understand that as the warranty increases, the manufacturer has to cover more warranty costs. Consequently, the expected manufacturer profit $\mathbb{E}\left(T P^{s}\right)$ and the expected cost of the consumer $\mathbb{E}\left(C^{s}\right)$ both decrease as the warranty period grows.

In presence of interaction between components, for short warranty period, the manufacturer profit increases. As the warranty period gets longer, the average total manufacturer benefit (or the consumer total average cost) decreases. This means that in the presence of interaction between components, a long warranty period is more cost efficient for the consumer. Indeed, the component dependency has a stronger impact on the long term where due to the aging of component 1 more and more maintenance is required. The component or system failure usually occurs after the warranty period resulting in a maintenance cost to the consumer and so a profit to the manufacturer. When $W$ gets larger, the failure interaction between components accelerates the failure within warranty leading to a gratis system maintenance for the consumer which decreases the income of the manufacturer. Therefore, in presence of dependence, the manufacturer profit (consumer cost) decreases for long warranty periods.

The impact of the repair degree of component 1

Figure 6 shows the expected manufacturer cost within the warranty period under RFRW with different repair degree of component 1 ( $a=0.2, a=0.6$ 


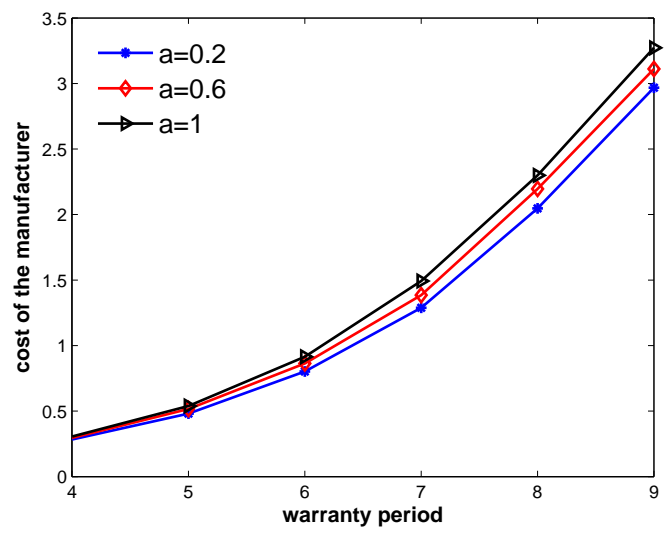

Figure 6: The expected manufacturer cost $\mathbb{E}\left(C^{R}(W)\right)$ under RFRW with different repair degree of component $1(a=0.2, a=0.6$ and $a=1)$.

and $a=1$ ). It shows that the manufacturer warranty cost increases with $a$. The larger $a$ is, the worse is the repair of component 1. More damages are induced to component 2 which accelerates its degradation and so its failure. Therefore more system maintenance and replacement costs are induced to the manufacturer expenses. It is noted that the manufacturer cost is less sensitive when $W$ is small. This is due to the fact that in our example, the occurrence of component 1 failure and the system failure are rare within small $W$, and component 1 is minimally repaired at failure.

In sum, it is more costly to the manufacturer to replace the product than to repair it. However, the cost difference is not significant and it could be profitable for its commercial image to propose a prefect or quasi perfect repair.

In the following, we reset $S=\{W=5, \lambda=8, b=1, a=1, \mu=5, \alpha=$ $\left.4, \beta=2, L=20, c_{11}=5, c_{22}=25, c_{1}=1, c_{2}=4, T=20\right\}$.

The impact of component 1 quality

Figure 7 shows that when the warranty period is small, both consumer cost and manufacturer profit decreases with $\lambda$. The smaller is $\lambda$, the shorter is the lifetime of component 1 . Therefore more component/system failure are occurred within the service time $T$. Hence for short warranty period, the consumer pays more and the manufacturer loses less. On the contrary, when the warranty period $W$ is large, during the period $T$, more failure costs are covered by the manufacturer which decreases its profit and the consumer 
cost.
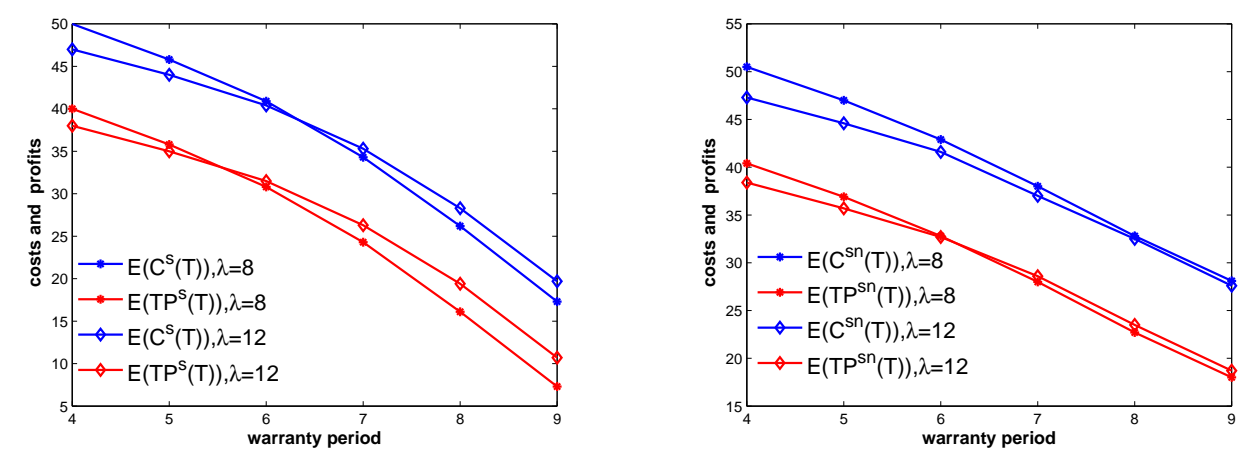

Figure 7: The expected consumer costs and manufacturer profits with different $\lambda(\lambda=8$, $\lambda=12)$.

The impact of component 2 natural deterioration

It is expected that as component 2 deteriorates faster, it is more beneficial for the consumer to have a warranty and more costly for the manufacturer to fulfill this option. This fact is highlighted in Figure 8. Similarly, it can be noticed in Figure 8 that the manufacturer profit and the consumer cost increase with $\alpha$ when $W$ is small and decrease with $\alpha$ when $W$ is large. With large $\alpha$, component 2 deteriorates faster and its lifetime is shorter. It is then more beneficial to the consumer to have long warranty period. For a short warranty period with these parameters setting, $\alpha \in\{4,6\}$, the failure is not mainly due to the natural deterioration, henceforth the total maintenance cost is not very sensitive to the changes of $\alpha$.

From the sensitivity analysis of the impacts of component 1 and component 2 , it is noticed that the initial warranty period $W$ has impact on the manufacturer's and the consumer's product quality preferences.

The impact of maintenance prices and costs

The manufacture profits and consumer costs under both RFRW and nonrenewing FRW with different maintenance prices and costs are presented in Tables 2 and 3 .

As expected, both the manufacturer profit and the consumer cost decrease with the value of $W$. As $W$ gets larger, more maintenance costs are covered by the manufacturer which induces the manufacture profit and the consumer cost. Both the consumer cost and the manufacturer profit increase with the 

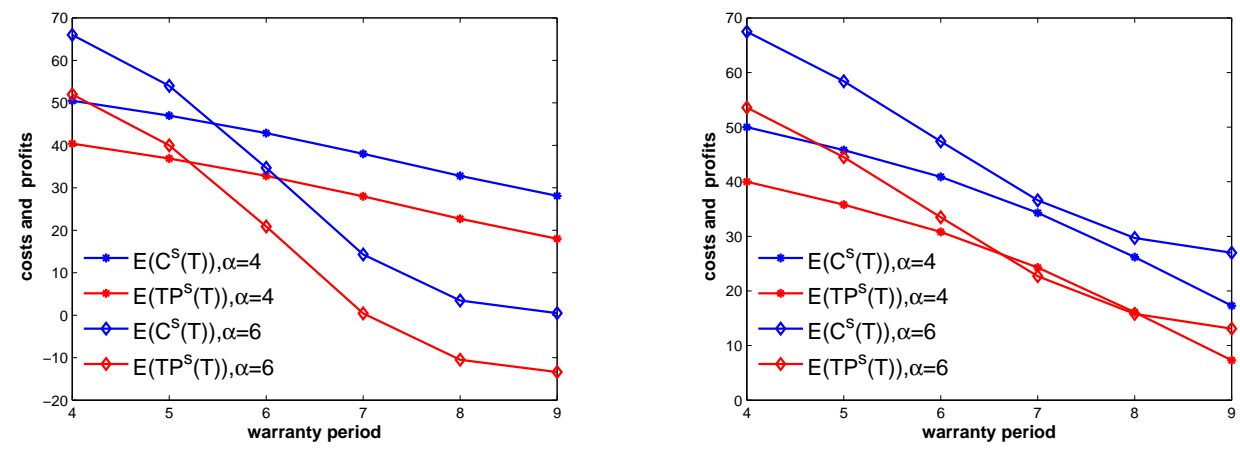

Figure 8: The expected consumer cost and manufacturer profits with different $\alpha(\alpha=4$, $\alpha=6)$.

\begin{tabular}{|c|c|c|c|c|c|c|c|c|c|}
\hline parameters & \multicolumn{3}{|c|}{$c_{11}=5, c_{22}=25$} & \multicolumn{3}{c|}{$c_{11}=5, c_{22}=15$} & \multicolumn{3}{c|}{$c_{11}=10, c_{22}=25$} \\
\cline { 2 - 10 } cost/profit & $W=4$ & $W=6$ & $W=9$ & $W=4$ & $W=6$ & $W=9$ & $W=4$ & $W=6$ & $W=9$ \\
\hline $\mathbb{E}\left(C^{s}(T)\right)$ & 50.0 & 40.9 & 17.3 & 31.5 & 25.0 & 10.5 & 53.6 & 42.5 & 17.5 \\
$\mathbb{E}\left(T P^{s}(T)\right)$ & 40.0 & 30.8 & 7.3 & 21.5 & 15.0 & 0.45 & 43.6 & 32.4 & 7.5 \\
$\mathbb{E}\left(C^{s n}(T)\right)$ & 50.5 & 42.9 & 28.1 & 29.6 & 25.6 & 17.0 & 51.3 & 43.3 & 29.0 \\
$\mathbb{E}\left(T P^{s n}(T)\right)$ & 40.4 & 32.8 & 18.0 & 20.6 & 16.7 & 8.2 & 41.3 & 34.3 & 20.1 \\
\hline
\end{tabular}

Table 2: The warranty costs and profits under the RFRW and the non-renewing FRW with different values of $c_{11}$ and $c_{22}$

consumers maintenance prices $c_{11}$ and $c_{22}$. Since $c_{1}$ and $c_{2}$ are maintenance costs for the manufacturer, the consumer maintenance cost is unchanged with $c_{1}$ and $c_{2}$. However, the manufacturer profits under both the RFRW and non-renewing FRW decrease as $c_{1}$ and $c_{2}$ increase.

It is obvious that the RFRW is more economic for the consumer and the non-renewing FRW is more favorable to the manufacturer. In addition, the most profitable policy for the manufacturer is no warranty policy under

\begin{tabular}{|c|c|c|c|c|c|c|c|c|c|}
\hline \multirow{2}{*}{$\begin{array}{c}\text { parameters } \\
\text { cost/profit }\end{array}$} & \multicolumn{3}{|c|}{$c_{1}=1, c_{2}=4$} & \multicolumn{3}{c|}{$c_{1}=1, c_{2}=8$} & \multicolumn{3}{c|}{$c_{1}=3, c_{2}=8$} \\
\cline { 2 - 10 } & $W=4$ & $W=6$ & $W=9$ & $W=4$ & $W=6$ & $W=9$ & $W=4$ & $W=6$ & $W=9$ \\
\hline $\mathbb{E}\left(C^{s}(T)\right)$ & 50.0 & 40.9 & 17.3 & 50.0 & 40.9 & 17.3 & 50.0 & 40.9 & 17.3 \\
$\mathbb{E}\left(T P^{s}(T)\right)$ & 40.0 & 30.8 & 7.3 & 31.7 & 22.7 & -1.0 & 27.9 & 18.6 & -4.7 \\
$\mathbb{E}\left(C^{s n}(T)\right)$ & 50.5 & 42.9 & 28.1 & 50.5 & 42.9 & 28.1 & 50.5 & 42.9 & 28.1 \\
$\mathbb{E}\left(T P^{s n}(T)\right)$ & 40.4 & 32.8 & 18.0 & 32.2 & 24.8 & 10.0 & 28.3 & 20.6 & 7.6 \\
\hline
\end{tabular}

Table 3: The warranty costs and profits under the RFRW and the non-renewing FRW with different values of $c_{1}$ and $c_{2}$ 
which the consumer covers the whole cost during its usage.

It can be observed in Table 4 that, in general, the non-renewing FRW policy incurs more significant consumer cost and manufacturer benefit. Indeed in this case, the manufacturer covers less maintenance under FRW and so the consumer cost increases in this policy. In our example, when there is no failure interaction between components and the warranty period $W$ is short, there is not a substantial difference between the RFRW and non-renewing FRW policy. Under the same parameters setting, the cost rate and the profit for the consumer and the manufacturer without warranty are $\mathbb{E}\left(C^{l}\right)=1.3$, $\mathbb{E}\left(T P^{l}\right)=6.4$ when $\mu=5$ and $\mathbb{E}\left(C^{l}\right)=1.4, \mathbb{E}\left(T P^{l}\right)=6.2$ when $\mu=0$ respectively.

\begin{tabular}{|c|c|c|c|c|c|c|}
\hline parameters & \multicolumn{3}{|c|}{$\mu=5$} & \multicolumn{3}{c|}{$\mu=0$} \\
\cline { 2 - 7 } cost/profit & $W=4$ & $W=6$ & $W=9$ & $W=4$ & $W=6$ & $W=9$ \\
\hline $\mathbb{E}\left(C^{r}\right)$ & 3.1 & 2.6 & 1.2 & 2.9 & 2.7 & 1.9 \\
$\mathbb{E}\left(T P^{r}\right)$ & 2.5 & 2.0 & 0.7 & 2.3 & 2.2 & 1.4 \\
$\mathbb{E}\left(C^{n r}\right)$ & 3.1 & 2.7 & 2.0 & 2.9 & 2.7 & 2.2 \\
$\mathbb{E}\left(T P^{n r}\right)$ & 2.5 & 2.1 & 1.4 & 2.3 & 2.2 & 1.7 \\
\hline
\end{tabular}

Table 4: The average cost rate and profit rate under RFRW and the non-renewing FRW policies with different values of $W$ and $\mu$

It is worth mentioning that the estimation of the warranty costs/profits to the consumer/manufacturer under different warranty policies could be references paving the way for lucrative market strategies. The important thing is the overall interest to the manufacturer rather then the profits/costs induced by the warranty individual.

\begin{tabular}{|c|cc|cc|}
\hline \multirow{2}{*}{ W and $\mathbf{T}$} & \multicolumn{2}{|c|}{ manufacturer's profit rate } & \multicolumn{2}{c|}{ consumer's cost rate } \\
\cline { 2 - 5 } & $\mathbb{E}\left(\mathbf{T P}^{\mathbf{s}}(\mathbf{T})\right) / \mathbf{T}$ & $\mathbb{E}\left(\mathbf{T P}^{\mathbf{s n}}(\mathbf{T})\right) / \mathbf{T}$ & $\mathbb{E}\left(\mathbf{C}^{\mathbf{S}}(\mathbf{T})\right) / \mathbf{T}$ & $\mathbb{E}\left(\mathbf{C}^{\mathbf{s n}}(\mathbf{T})\right) / \mathbf{T}$ \\
\hline$(\mathbf{W}, \mathbf{T})=(4,20)$ & $\mathbf{2 . 0 0}$ & $\mathbf{2 . 0 2}$ & $\mathbf{2 . 5 0}$ & $\mathbf{2 . 5 3}$ \\
\hline$(\mathbf{W}, \mathbf{T})=(6,20)$ & 1.54 & 1.64 & 2.05 & 2.15 \\
\hline$(\mathbf{W}, \mathbf{T})=(6, \infty)$ & 2.00 & 2.10 & 2.60 & 2.70 \\
\hline
\end{tabular}

Table 5: The average cost rate and profit rate in the long-run horizon and in the short-run horizon respectively

The comparison between long-run and short-run Table 5 presents the comparison of the short-run and long-run cost rates and profit rates form the points of view of the consumer and the manufacturer respectively. It is observed that, under both the 
non-renewing FRW and the RFRW, the profit rate of the manufacture show an decreasing tendency with respect to the warranty period $W$. On the contrary, the consumer spends less as more maintenance costs are covered by the manufacturer. Besides, the long-run profit rate of the manufacturer is higher than that in the short run. Therefore the manufacturer may underestimate their benefit if they take the long-run profit rate as a criterion when the product service time is short-term.

\section{Conclusions}

In this study, a warranty cost and profit model for a two-component series system with failure interactions is developed. By considering the product service time to the consumer, the warranty cost and profit from both point of view, the manufacturer and the consumer, are derived. The exact numerical results and Monte Carlo simulations are presented. It is shown that the failure interaction between components can affect the system lifetime and therefore the warranty cost and the profit. The initial warranty length has impact on the manufacturer's and the consumer's product quality preferences. The warranty length is established by the manufacturer based on the warranty cost/profit, as well as system reliability, and is intended to maximize the manufacturer's profit. Therefore it would be beneficial to the manufacturer to mitigate the stochastic dependence between components in product design phase. It is recommended to the decision-maker to consider the warranty cost budget in the develop of the warranty strategy, to take the warranty cost as a reference in the evaluation of the product profit, etc.

In our future works, we intend to consider the warranty cost analysis of more complex systems with different structure configuration, warranty policies, maintenance types, etc.

[1] Babishin, V., Taghipour, S.. Optimal maintenance policy for multicomponent systems with periodic and opportunistic inspections and preventive replacements. Applied Mathematical Modelling 2016;40(23):1048010505.

[2] Bai, J., Pham, H.. Discounted warranty cost of minimally repaired series systems. IEEE Transactions on Reliability 2004;53(1):37-42. 
[3] Caballé, N., Castro, I.. Analysis of the reliability and the maintenance cost for finite life cycle systems subject to degradation and shocks. Applied Mathematical Modelling 2017;52:731-746.

[4] Chen, T., Popova, E.. Maintenance policies with two-dimensional warranty. Reliability Engineering \& System Safety 2002;77(1):61-69.

[5] Chien, Y.H.. Optimal age for preventive replacement under a combined fully renewable free replacement with a pro-rata warranty. International Journal of Production Economics 2010;124(1):198-205.

[6] Glickman, T.S., Berger, P.D.. Optimal price and protection period decisions for a product under warranty. Management Science 1976;22(12):1381-1390.

[7] Jack, N., Dagpunar, J.. An optimal imperfect maintenance policy over a warranty period. Microelectronics Reliability 1994;34(3):529-534.

[8] Jain, M., Maheshwari, S.. Discounted costs for repairable units under hybrid warranty. Applied Mathematics and Computation 2006;173(2):887-901.

[9] Jung, K.M., Park, M., Park, D.H.. Optimal maintenance strategy for non-renewing replacement-repair warranty. Applied Stochastic Models in Business and Industry 2012;28(6):607-614.

[10] Kijima, M.. Some results for repairable systems with general repair. Journal of Applied probability 1989;26(1):89-102.

[11] Kijima, M., Morimura, H., Suzuki, Y.. Periodical replacement problem without assuming minimal repair. European Journal of Operational Research 1988;37(2):194-203.

[12] Liu, B., Wu, J., Xie, M.. Cost analysis for multi-component system with failure interaction under renewing free-replacement warranty. European Journal of Operational Research 2015;243(3):874-882.

[13] Mamer, J.W.. Cost analysis of pro rata and free-replacement warranties. Naval Research Logistics Quarterly 1982;29(2):345-356. 
[14] Murthy, D., Djamaludin, I.. New product warranty: A literature review. International Journal of Production Economics 2002;79(3):231260.

[15] Murthy, D., Nguyen, D.. Study of a multi-component system with failure interaction. European Journal of Operational Research 1985;21(3):330-338.

[16] Murthy, D., Nguyen, D.. Study of two-component system with failure interaction. Naval Research Logistics Quarterly 1985;32(2):239-247.

[17] Nakagawa, T.. Stochastic processes: With applications to reliability theory. Springer Science \& Business Media, 2011.

[18] Nakagawa, T., Murthy, D.. Optimal replacement policies for a twounit system with failure interactions. Revue française d'automatique, d'informatique et de recherche opérationnelle Recherche opérationnelle 1993;27(4):427-438.

[19] Nguyen, D., Murthy, D.. Cost analysis of warranty policies. Naval Research Logistics Quarterly 1984;31(4):525-541.

[20] Park, M., Jung, K.M., Park, D.H.. Optimal maintenance strategy under renewable warranty with repair time threshold. Applied Mathematical Modelling 2017;43:498-508.

[21] Park, M., Pham, H.. Warranty cost analyses using quasi-renewal processes for multicomponent systems. IEEE Transactions on Systems, Man, and Cybernetics-Part A: Systems and Humans 2010;40(6):13291340 .

[22] Park, M., Pham, H.. Cost models for age replacement policies and block replacement policies under warranty. Applied Mathematical Modelling 2016;40(9):5689-5702.

[23] Satow, T., Osaki, S.. Optimal replacement policies for a two-unit system with shock damage interaction. Computers \& Mathematics with Applications 2003;46(7):1129-1138.

[24] Sheu, S.H., Chen, Y.L., Chang, C.C., Zhang, Z.G.. A note on a two variable block replacement policy for a system subject to 
non-homogeneous pure birth shocks. Applied Mathematical Modelling 2016;40(5):3703-3712.

[25] Sheu, S.H., Sung, C.K., Hsu, T.S., Chen, Y.C.. Age replacement policy for a two-unit system subject to non-homogeneous pure birth shocks. Applied Mathematical Modelling 2013;37(10):7027-7036.

[26] Van Noortwijk, J.. A survey of the application of gamma processes in maintenance. Reliability Engineering \& System Safety 2009;94(1):2-21.

[27] Wang, G.J., Yam, R.C.. Generalized geometric process and its application in maintenance problems. Applied Mathematical Modelling 2017;49:554-567.

[28] Wang, G.J., Zhang, Y.L.. A bivariate mixed policy for a simple repairable system based on preventive repair and failure repair. Applied Mathematical Modelling 2009;33(8):3354-3359.

[29] Wu, C.C., Chou, C.Y., Huang, C.. Optimal burn-in time and warranty length under fully renewing combination free replacement and pro-rata warranty. Reliability Engineering \& System Safety 2007;92(7):914-920.

[30] Wu, S., Longhurst, P.. Optimising age-replacement and extended nonrenewing warranty policies in lifecycle costing. International Journal of Production Economics 2011;130(2):262-267.

[31] Yu, H., Chu, C., Châtelet, É.. Availability optimization of a redundant system through dependency modeling. Applied Mathematical Modelling 2014;38(19):4574-4585.

[32] Yun, W.Y., Lee, Y.W., Ferreira, L.. Optimal burn-in time under cumulative free replacement warranty. Reliability Engineering \& System Safety 2002;78(2):93-100.

[33] Zong, S., Chai, G., Zhang, Z.G., Zhao, L.. Optimal replacement policy for a deteriorating system with increasing repair times. Applied Mathematical Modelling 2013;37(23):9768-9775. 